\title{
An unusual case of a persistent, infected retroperitoneal fluid collection 5 years after anterior lumbar fusion surgery: illustrative case
}

\author{
Matthew T. Neal, MD, ${ }^{1}$ Kara L. Curley, PA-C, ${ }^{1}$ Alexandra E. Richards, DNP, FNP-BC, ${ }^{1}$ Maziyar A. Kalani, MD, ${ }^{1}$ Mark K. Lyons, MD, ${ }^{1}$ and \\ Victor J. Davila, MD²
}

Departments of ${ }^{1}$ Neurologic Surgery and ${ }^{2}$ Vascular Surgery, Mayo Clinic, Phoenix, Arizona

\begin{abstract}
BACKGROUND Anterior lumbar fusion procedures have many benefits and continue to grow in popularity. The technique has many potential approach- and procedure-related complications. Symptomatic retroperitoneal fluid collections are uncommon but potentially serious complications after anterior lumbar procedures. Collection types include hematomas, urinomas, chyloperitoneum, cerebrospinal fluid collections, and deep infections.

OBSERVATIONS The authors present an unusual case of a patient with persistent symptoms related to a retroperitoneal collection over a 5 -year period following anterior lumbar fusion surgery. To the authors' knowledge, no similar case with such extensive symptom duration has been described. The patient had an infected encapsulated fluid collection. The collection was presumed to be a postoperative lymphocele that was secondarily infected after serial percutaneous drainage procedures.
\end{abstract}

LESSONS When retroperitoneal collections occur after anterior retroperitoneal approaches, clinical clues, such as timing of symptoms, hypotension, acute anemia, urinary tract infection, hydronephrosis, elevated serum creatinine and blood urea nitrogen, low-pressure headaches, anorexia, or systemic signs of infection, can help narrow the differential. Retroperitoneal collections may continue to be symptomatic many years after anterior lumbar surgery. The collections may become infected after serial percutaneous drainage or prolonged continuous drainage. Encapsulated, infected fluid collections typically require surgical debridement of the capsule and its contents.

https://thejns.org/doi/abs/10.3171/CASE20107

KEYWORDS anterior lumbar fusion; ALIF; spinal infection; chyloperitoneum; lymphocele

The anterior lumbar interbody fusion (ALIF) is a well-established surgical technique and is routinely used by many spine surgeons. ${ }^{1}$ The indications for ALIF are diverse and include degenerative, deformityrelated, iatrogenic, infectious, and oncological pathologies. ${ }^{2-6}$ The procedure has many advantages, such as avoidance of direct manipulation of the neural elements, indirect neural decompression, ${ }^{7-9}$ high fusion rates in excess of $95 \%, 2,10,11$ and significant improvement in desired sagittal radiographic parameters. ${ }^{12}$ In addition, the ALIF technique has been associated with significant improvement in commonly used patient-reported outcome measures, including the total visual analog scale, Oswestry Disability Index, and Scoliosis Research Society-22 scale., ${ }^{9,11-14}$

Overall, the complication rates for ALIF are relatively low. A recent meta-analysis on complications in anterior lumbar surgery that included 76 articles and 11,410 collective cases revealed a complication rate of $14.1 \%$, including minor and major complications. The most common complications, such as venous injury and retrograde ejaculation, occurred less than $3 \%$ of the time. ${ }^{15}$

Other ALIF complications that have been described include ureter injury, neurological injury, postoperative ileus, colonic pseudoobstruction, superficial infection, urinary tract infection, sepsis, subsidence, pseudarthrosis, instrumentation failure, adjacent segment disease, sacral fractures, and retroperitoneal fluid collections. ${ }^{10,16-24}$ Retroperitoneal fluid collections are an uncommon cause of delayed symptoms following an ALIF procedure. Various etiologies include hematomas, urinomas, chyloperitoneum, cerebrospinal fluid (CSF) collections, and deep infections.

We present an unusual case with a contained, retroperitoneal, infected fluid collection in a patient 5 years after an ALIF procedure. Management and key learning points are discussed. To our knowledge, no similar case has been described.

ABBREVIATIONS ALIF = anterior lumbar interbody fusion; CSF = cerebrospinal fluid; CT = computed tomography; MRI = magnetic resonance imaging. INCLUDE WHEN CITING Published January 25, 2021; DOI: 10.3171/CASE20107.

SUBMITTED November 12, 2020. ACCEPTED November 20, 2020.

(C) 2021 The authors, CC BY-NC-ND 4.0 (http://creativecommons.org/licenses/by-nc-nd/4.0/), 


\section{Illustrative Case}

A 71-year-old male with a history of obesity, type 2 diabetes mellitus, prostate cancer status post resection and radiation, and an L4-5 and L5-S1 ALIF procedure 5 years earlier presented with abdominal discomfort and fullness that had been present since the ALIF procedure. He had intermittent episodes of sharp, left-sided abdominal pain. Over several years, he had undergone multiple aspiration procedures at outside medical facilities to drain the retroperitoneal fluid collection. Unfortunately, fluid analysis reports were not available for review. He denied acute low-back pain, fever, lower extremity pain, or other acute neurological symptoms at the time of presentation.

On examination, he was a well-developed male who appeared his stated age. He had a well-healed paramedian surgical incision on his left abdomen consistent with the prior ALIF approach. There was no erythema surrounding the incision; however, there was some fullness in the left lower quadrant and minimal abdominal tenderness was noted.

An abdominal magnetic resonance imaging (MRI) study was obtained that revealed a $7.6 \times 12.1 \times 12.1-\mathrm{cm}$ retroperitoneal fluid collection with mixed signal in the left lower quadrant of the abdomen (Fig. 1). The collection was in the same plane as the prior retroperitoneal ALIF approach, although there was no direct communication with the spinal hardware. There was faint enhancement around the periphery of the collection and necrotic-appearing debris along the posterior margin. There were no abnormalities with other abdominal organs. Specifically, there was no hydronephrosis, pancreatitis, cirrhosis, lymphadenopathy, intestinal abnormality, or vascular abnormality.

Serum laboratory analysis demonstrated a normal leukocyte count $\left(6.4 \times 10^{9} / \mathrm{L}\right)$ and normal values on basic metabolic panel.

A computed tomography (CT)-guided drain was placed, and fluid was sent for analysis. The hazy, red fluid contained 5,556 nucleated cells $/ \mu \mathrm{L}$ (87\% neutrophils). A Gram stain revealed rare white blood cells, and bacterial cultures were negative. Triglycerides were $234 \mathrm{mg} / \mathrm{dL}$, although laboratory notes commented that the specimen was hemolyzed, which could interfere with test results. Several days

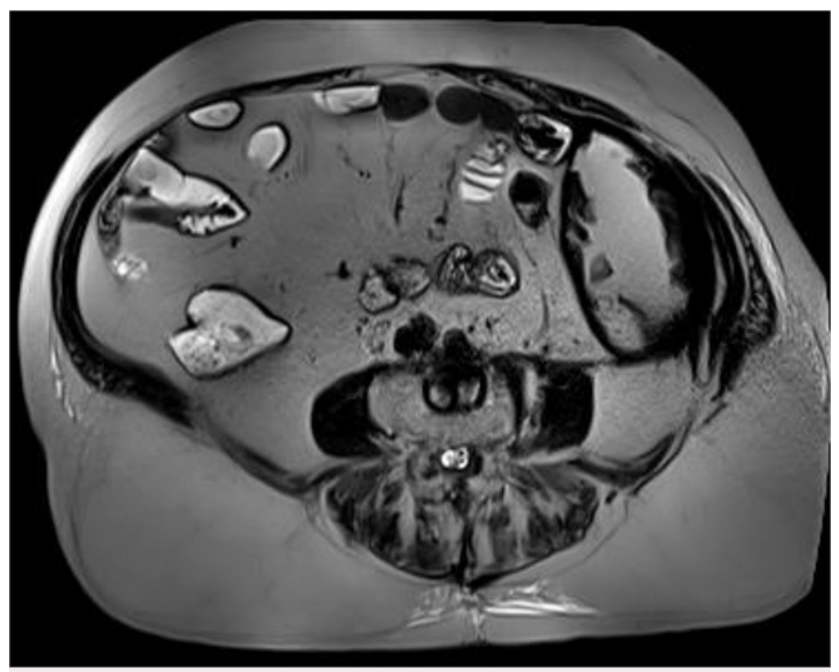

FIG. 1. T2 MRI demonstrating a 12-cm left-sided retroperitoneal fluid collection with mixed signal. later, CT imaging showed complete drainage of the fluid cavity. A sclerotherapy procedure was performed using doxycycline prior to drain removal.

A follow-up CT scan of the abdomen approximately 2 months later demonstrated recurrence and enlargement of the abdominal fluid collection (Fig. 2). The patient was subsequently taken for open surgical debridement. Intraoperatively, a thickened, well-organized capsule surrounding the fluid collection was encountered in the lateral extraperitoneal space. The capsule was incised sharply, resulting in immediate evacuation of a large amount of thin, beige-colored, opaque fluid, which was sent for analysis. The cavity contained a large amount of semisolid, necrotic material, and no frank purulent material (Fig. 3). The cavity did not communicate with the peritoneum or the spinal hardware. A thorough debridement was performed and all necrotic material was removed. A large-caliber closed suction drain was left in place. The drain was maintained for 10 days to ensure daily output was less than $30 \mathrm{ml}$. Gram stains were positive for gram-positive cocci, and aerobic bacterial culture grew Staphylococcus epidermis. Remaining cultures and beta-2 transferrin testing were negative. After susceptibility testing for the bacterium, the patient was treated with a 10-day course of Augmentin (Mylan Pharmaceuticals), $875 \mathrm{mg}$ twice daily.

Follow-up CT imaging 6 months later demonstrated minimal residual, benign-appearing fluid in the retroperitoneal plane (Fig. 4). The patient reported resolution of abdominal pain and discomfort. He had no signs or symptoms of local or systemic infection.

\section{Discussion}

\section{Observations}

Secondary to the many benefits of ALIF surgery, the number of ALIF procedures performed in the United States has continued to grow steadily. 1,12 Therefore, it is important for surgeons performing these procedures to be aware of potential complications. One possible complication is a retroperitoneal fluid collection. Potential types of

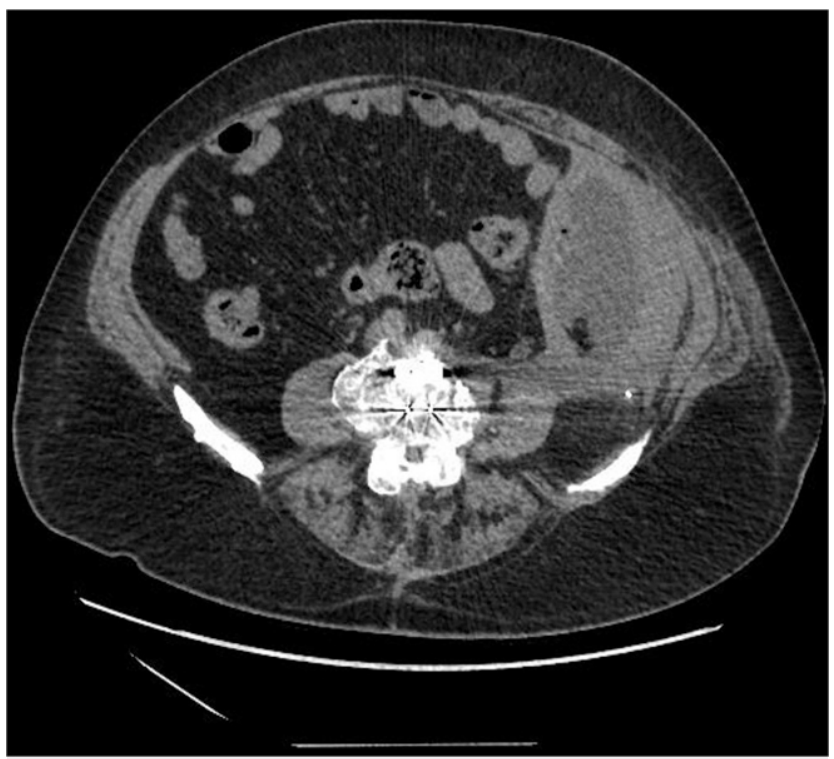

FIG. 2. CT demonstrating recurrence of a left-sided retroperitoneal fluid collection after percutaneous drainage. 


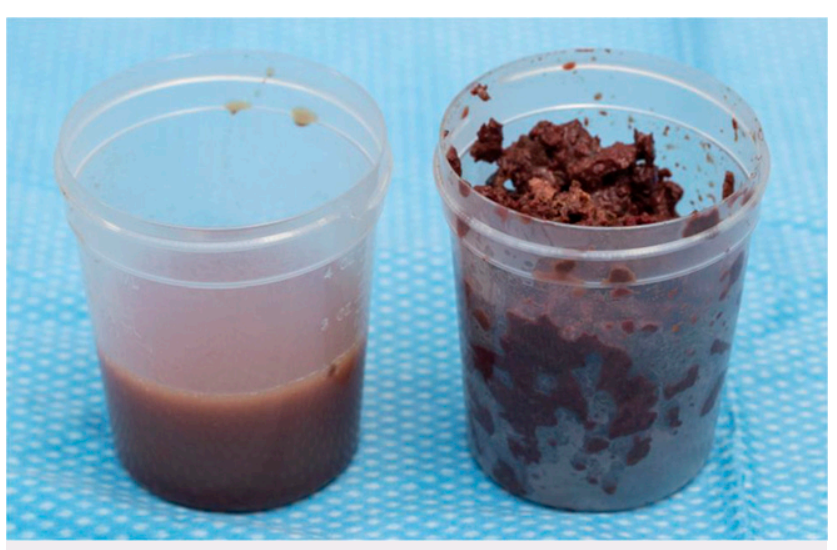

FIG. 3. Cystic fluid and internal necrotic debris collected during open debridement.

retroperitoneal fluid collections include hematoma; CSF collection; urinoma; chyloperitoneum; and encapsulated, infected fluid collection. 23,25-30 We suspect the patient in this case had a lymphocele that was secondarily infected following his ALIF. Lymphoceles can result from dissection of the aorta and iliac vessels, causing disruption of lymphatic vessels. The lymphatic leak was probably unrecognized because lymphatic flow can be as slow as $1 \mathrm{ml} / \mathrm{min}$. ${ }^{26,31}$ Development of a symptomatic lymphocele after spine surgery is uncommon and is more frequently reported after transplant or major gynecological surgery. ${ }^{32,33}$ Nonetheless, symptomatic lymphoceles can occur after anterior lumbar surgery, and a recent review article identified 33 cases in the literature. ${ }^{26}$

Because symptomatic lymphatic collections after anterior lumbar surgery are rare, management strategies are not well described in the literature. The previously mentioned review article proposes a management protocol for chyloperitoneum after anterior lumbar surgery. ${ }^{26}$ The authors endorse prolonged percutaneous drainage in conjunction with sclerosing agents such as doxycycline, bleomycin, OK-432, povidone-iodine, and ethiodized oil. The use of sclerosing agents likely shortens the necessary duration of drainage. ${ }^{34}$ Refractory cases require laparoscopic fenestration, allowing drainage into the peritoneum, or open surgical treatment. ${ }^{26}$

Following a systematic approach, we were able to successfully manage the symptomatic retroperitoneal collection in this case. Based on the patient's history and examination, CT and MRI scans were obtained to rule out pathologies other than the fluid collection, which was suspected based on prior history. For diagnostic and therapeutic purposes, percutaneous drainage and injection of a sclerosing agent were performed. Fluid specimens were analyzed with cell counts, triglycerides, and cultures for infectious agents. ${ }^{26,27}$ If urinoma had been suspected, the fluid's creatinine and urea would have been analyzed and compared to serum levels. When the fluid collection recurred, we performed open debridement of the thick capsule and its contents. In this case, infection was identified after open debridement, and antibiotics were administered for definitive treatment.

\section{Lessons}

We believe there are several aspects of this case that are instructive. When retroperitoneal collections occur after anterior retroperitoneal approaches, clinical clues, such as timing of symptoms, hypotension, acute anemia, urinary tract infection, hydronephrosis, elevated serum creatinine and blood urea nitrogen, low-pressure

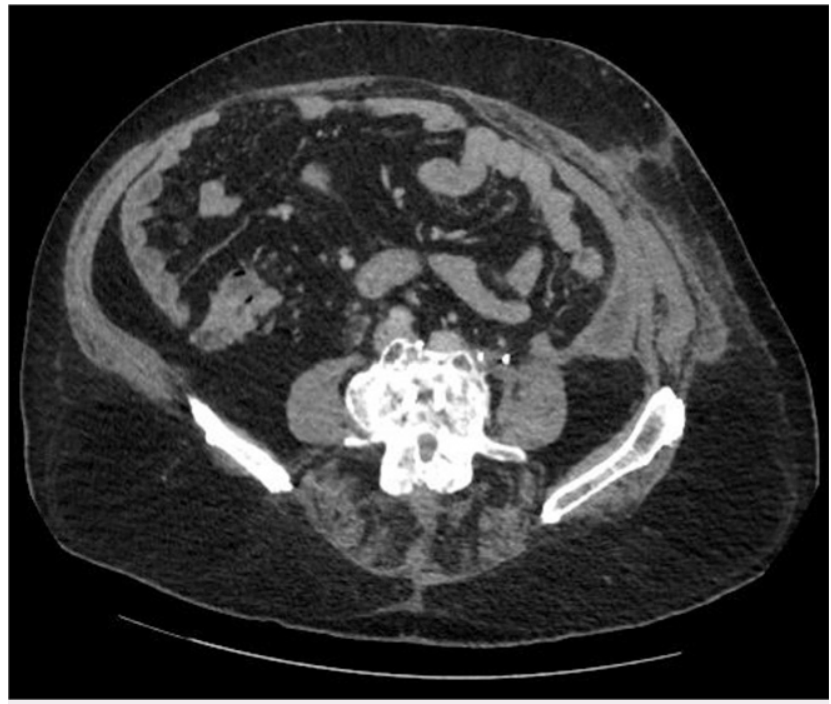

FIG. 4. CT of the abdomen 6 months after surgery revealing a minimal residual retroperitoneal fluid collection.

headaches, anorexia, or systemic signs of infection, can help narrow the differential. Retroperitoneal collections may continue to be symptomatic many years after ALIF surgery. To our knowledge, no similar case with such extensive symptom duration has been described. Furthermore, a recent review analyzing 33 published cases of chyloperitoneum after anterior lumbar surgery did not identify any cases with secondary infections after drainage procedures ${ }^{26}$ Clinicians should consider that retroperitoneal fluid collections may become infected after serial percutaneous drainage or prolonged continuous drainage. Also, clinicians must maintain suspicion for infection, despite false-negative culture results after percutaneous drainage. Encapsulated, infected fluid collections typically require surgical debridement of the capsule and its contents.

\section{References}

1. Varshneya $K$, Medress ZA, Jensen M, et al. Trends in anterior lumbar interbody fusion in the United States: a MarketScan study from 2007 to 2014. Clin Spine Surg. 2020;33(5):E226-E230.

2. Kalb S, Perez-Orribo L, Kalani MYS, et al. The influence of common medical conditions on the outcome of anterior lumbar interbody fusion. Clin Spine Surg. 2016;29(7):285-290.

3. Chang CW, Fu TS, Chen WJ, et al. Management of infected transforaminal lumbar interbody fusion cage in posterior degenerative lumbar spine surgery. World Neurosurg. 2019;126: e330-e341.

4. Lyons MK, Neal MT, Patel NP, et al. Progressive back pain due to Aspergillus nidulans vertebral osteomyelitis in an immunocompetent patient: surgical and antifungal management. Case Rep Orthop. 2019;2019:4268468.

5. Lin Y, Li F, Chen W, et al. Single-level lumbar pyogenic spondylodiscitis treated with mini-open anterior debridement and fusion in combination with posterior percutaneous fixation via a modified anterior lumbar interbody fusion approach. J Neurosurg Spine. 2015;23(6):747-753.

6. Matsui H, Hirano N, Sakaguchi Y. Vertebral osteomyelitis: an analysis of 38 surgically treated cases. Eur Spine J. 1998;7(1):50-54.

7. Bozzio $A E$, Johnson CR, Fattor JA, et al. Stand-alone anterior lumbar interbody, transforaminal lumbar interbody, and anterior/ 
posterior fusion: analysis of fusion outcomes and costs. Orthopedics. 2018;41(5):e655-e662.

8. Khan HA, Stumpf NJ, Isbester KA, et al. Anterior lumbar interbody fusion reduces thecal sac compression in the setting of degenerative spondylolisthesis. Spine J. 2020;20(7):1035-1036.

9. Kapustka B, Kiwic G, Chodakowski P, et al. Anterior lumbar interbody fusion (ALIF): biometrical results and own experiences. Neurosurg Rev. 2020;43(2):687-693.

10. Manzur M, Virk SS, Jivanelli B, et al. The rate of fusion for standalone anterior lumbar interbody fusion: a systematic review. Spine J. 2019;19(7):1294-1301.

11. Szadkowski M, d'Astorg $\mathrm{H}$, Bouhali $\mathrm{H}$, et al. Outcomes of standalone anterior lumbar interbody fusion of L5-S1 using a novel implant with anterior plate fixation. Spine J. 2020;20(10): 1618-1628.

12. Hosseini $P$, Mundis GM Jr, Eastlack RK, et al. Preliminary results of anterior lumbar interbody fusion, anterior column realignment for the treatment of sagittal malalignment. Neurosurg Focus. 2017; 43(6): E6.

13. Kleimeyer JP, Cheng I, Alamin TF, et al. Selective anterior lumbar interbody fusion for low back pain associated with degenerative disc disease versus nonsurgical management. Spine (Phila Pa 1976). 2018;43(19):1372-1380.

14. Phan K, Thayaparan GK, Mobbs RJ. Anterior lumbar interbody fusion versus transforaminal lumbar interbody fusion--systematic review and meta-analysis. Br J Neurosurg. 2015;29(5):705-711.

15. Bateman DK, Millhouse PW, Shahi N, et al. Anterior lumbar spine surgery: a systematic review and meta-analysis of associated complications. Spine J. 2015;15(5):1118-1132.

16. Luu HY, Zobel MJ, Jonzzon S, et al. Predictors and outcomes of paramedian incisional hernia after anterior spine exposure. J Surg Res. 2020;247:380-386.

17. Ukogu CO, Jacobs S, Ranson WA, et al. Preoperative nutritional status as a risk factor for major postoperative complications following anterior lumbar interbody fusion. Global Spine J. 2018; 8(7):662-667.

18. Isiklar ZU, Lindsey RW, Coburn M. Ureteral injury after anterior lumbar interbody fusion. A case report. Spine (Phila Pa 1976). 1996; 21(20):2379-2382.

19. Rao PJ, Phan K, Giang G, et al. Subsidence following anterior lumbar interbody fusion (ALIF): a prospective study. J Spine Surg. 2017;3(2):168-175.

20. Lee CW, Yoon KJ, Ha SS. Which approach is advantageous to preventing development of adjacent segment disease? Comparative analysis of 3 different lumbar interbody fusion techniques (ALIF, LLIF, and PLIF) in L4-5 spondylolisthesis. World Neurosurg. 2017;105:612-622.

21. Lindley EM, McBeth ZL, Henry SE, et al. Retrograde ejaculation after anterior lumbar spine surgery. Spine (Phila Pa 1976). 2012; 37(20):1785-1789.

22. Lastfogel JF, Altstadt TJ, Rodgers RB, et al. Sacral fractures following stand-alone L5-S1 anterior lumbar interbody fusion for isthmic spondylolisthesis. J Neurosurg Spine. 2010;13(2):288-293.
23. VanValkenburg S, Trussell JC, Lavelle WF. Retroperitoneal fluid collection following anterior spine surgery--differential and management. Can J Urol. 2016;23(2):8243-8246.

24. Mahoney JR, Barnes D, Pahl D. Perforated ileus after L5-S1 anterior lumbar interbody fusion: a case report presentation. $A M E$ Case Rep. 2019;3:21

25. Gurgo RD, Turkiewicz DB, Guazzo EP, et al. A cerebrospinal fluid collection presenting as an abdominal mass following lumbar vertebrectomy for trauma. Aust NZ J Surg. 2000;70(2):140-141.

26. Joubert $C$, Monchal T, Junca-Laplace $C$, et al. Management of chyloretroperitoneum after lumbar surgery by anterior approach. World Neurosurg. 2019;122:e1211-e1221.

27. Hey HWD, Wong KL, Gatam AR, et al. Delayed lymphocele formation following lateral lumbar interbody fusion of the spine. Eur Spine J. 2017;26(1)(suppl 1):36-41.

28. Hussain NS, Hanscom D, Oskouian RJ Jr. Chyloretroperitoneum following anterior spinal surgery. J Neurosurg Spine. 2012;17(5): 415-421.

29. Manunga J, Alcala C, Smith J, et al. Technical approach, outcomes, and exposure related complications in patients undergoing anterior lumbar interbody fusion. J Vasc Surg. 2020. In press.

30. Gayer G, Caspi I, Garniek A, et al. Perirectal urinoma from ureteral injury incurred during spinal surgery mimicking rectal perforation on computed tomography scan. Spine (Phila Pa 1976). 2002; 27(20):E451-E453.

31. Hanson D, Mirkovic S. Lymphatic drainage after lumbar surgery. Spine (Phila Pa 1976). 1998;23(8):956-958.

32. Dodd GD, Rutledge F, Wallace S. Postoperative pelvic lymphocysts. Am J Roentgenol Radium Ther Nucl Med. 1970;108(2): 312-323

33. Ziêtek Z, Sulikowski T, Tejchman K, et al. Lymphocele after kidney transplantation. Transplant Proc. 2007;39(9):2744-2747.

34. Kerlan RK Jr, LaBerge JM, Gordon RL, et al. Bleomycin sclerosis of pelvic lymphoceles. J Vasc Interv Radiol. 1997;8(5):885-887.

\section{Disclosures}

The authors report no conflict of interest concerning the materials or methods used in this study or the findings specified in this paper

\section{Author Contributions}

Conception and design: Neal, Kalani, Davila. Acquisition of data: Neal, Curley, Kalani. Analysis and interpretation of data: Neal, Curley, Lyons. Drafting the article: Neal, Curley, Richards, Lyons. Critically revising the article: Neal, Curley, Richards, Lyons, Davila. Reviewed submitted version of manuscript: all authors. Approved the final version of the manuscript on behalf of all authors: Neal. Administrative/technical/ material support: Curley.

\section{Correspondence}

Matthew T. Neal: Mayo Clinic, Phoenix, AZ.neal.matthew@ mayo.edu. 